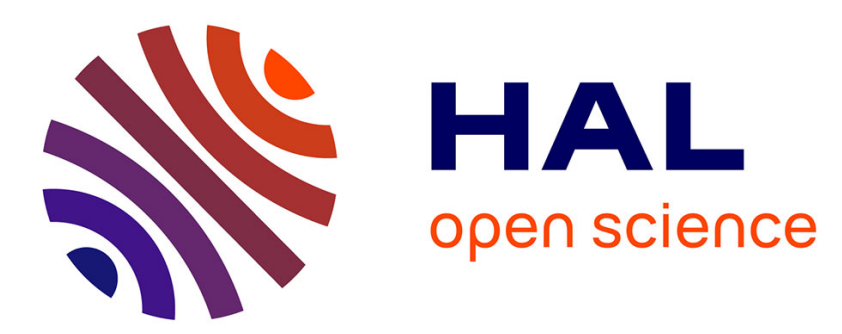

\title{
Formation flying control via elliptical virtual structure
}

\author{
Arthur Kahn, Julien Marzat, Hélène Piet-Lahanier
}

\section{To cite this version:}

Arthur Kahn, Julien Marzat, Hélène Piet-Lahanier. Formation flying control via elliptical virtual structure. IEEE International Conference on Networking, Sensing and Control, Apr 2013, Evry,

France. hal-00815705

\section{HAL Id: hal-00815705 \\ https://hal.science/hal-00815705}

Submitted on 19 Apr 2013

HAL is a multi-disciplinary open access archive for the deposit and dissemination of scientific research documents, whether they are published or not. The documents may come from teaching and research institutions in France or abroad, or from public or private research centers.
L'archive ouverte pluridisciplinaire HAL, est destinée au dépôt et à la diffusion de documents scientifiques de niveau recherche, publiés ou non, émanant des établissements d'enseignement et de recherche français ou étrangers, des laboratoires publics ou privés. 


\title{
Formation flying control via elliptical virtual structure
}

\author{
Arthur Kahn, Julien Marzat and Hélène Piet-Lahanier \\ ONERA - The French Aerospace Lab \\ F-91123 Palaiseau, France \\ Email: \{arthur.kahn, julien.marzat, helene.piet-lahanier\}@onera.fr
}

\begin{abstract}
This paper describes a guidance law for the formation flight of a flock of autonomous vehicles. The formation is defined by a virtual geometrical structure - here, an ellipse that can modify its shape and orientation to avoid collision with obstacles of the environment. The proposed guidance law is divided into two layers, with a model predictive control scheme at each level. The higher layer controls the structure itself to fulfill the goals and constraints of the required mission. The trajectory of the fleet is built on-line thanks to this layer, as well as the adaptation of the structure to the environment. The lower layer controls the vehicles, so as to attract and keep them inside the structure without side collision. Simulation results illustrate the interest of the approach to guide a fleet of vehicles towards a target while avoiding obstacles.
\end{abstract}

\section{INTRODUCTION}

Missions to be performed by unmanned aerial vehicles (UAV), such as area surveillance or exploration, can be addressed more efficiently by using multiple cooperating vehicles instead of a technically complex one. Formation flying is often sought in this context, since it allows large area coverage, drag reduction and maintaining communication range between vehicles. The topic of this paper is formation creation and guidance to a given target for a fleet of initially scattered vehicles, with obstacle avoidance. Three main types of methods can be found in the literature to address these issues, namely leader following, behavioural rules and virtual structure control.

In leader following, UAVs follow one member of the group defined as the leader. The leader has access to information that the other UAVs ignore, such as the trajectory to the destination of the fleet (see e.g. [1]-[6]). However, these approaches require to give more importance to one specific vehicle. The determination of the leader trajectory is a difficult balance between mission fulfillment and consistency with the movements of the flock. If a problem occurs on the leading vehicle, it becomes necessary to provide rule for exchanging hierarchical roles.

The second method of formation control is based on behavioural rules that each agent should follow according to its own task and the environment influence (see, e.g., [7][11]). Such approaches are nevertheless usually very problemdependent and are not easy to modify whenever an unexpected event occurs.

The third method uses virtual geometrical structures in which the agents should remain. The control law must first ensure that the agents are located within the structure and then define a suitable structure evolution depending on the mission requirements. In [12], all the agents define a structure similar to a rigid body. A control law moves this rigid body along a trajectory while trying to keep the formation stable. In [13], virtual structures are created with potential fields. Potential fields are also used in [14] to maintain the agents inside an elliptical ring area. The agents are allocated homogeneously inside this area. Sliding mode control is used in [15] to gather and maintain a set of agents inside an elliptical area. The area is arbitrarily moved while maintaining cohesion between the agents during the movement.

In this paper, the initially scattered UAVs are required to create a formation via a virtual structure and to reach a final destination. On the way to this target, the formation may encounter obstacles that it has to avoid. No off-line path planning is used to define trajectories bypassing the obstacles that are detected during the mission. The movement is assumed to be $2 \mathrm{D}$ and the UAVs are pointwise.

The guidance law is designed in order to move the virtual structure, chosen to be an ellipse, towards the destination while insuring that the UAVs remain inside. It consists of two layers. The first layer, described in Section II, uses a dynamical model for the guidance of the virtual structure with obstacle avoidance. The second layer, presented in Section III, is a decentralized control law that gathers the agents within the structure and prevents side collision. Model Predictive Control (MPC) is employed to design each of the two layers of the guidance law. Simulation results are presented in Section IV to illustrate the benefits of the proposed scheme.

\section{ViRTUAL STRUCTURE CONTROL}

The first layer of the guidance law generates the evolution of the virtual structure and adapts its shape so that it does not collide with the obstacles on its way to the target. It has been chosen to describe the formation shape as an ellipse, represented only by its center and characteristic matrix (this description can be steadily extended to that of an ellipsoid in 3D).

\section{A. Ellipse description}

An ellipse with center $\mathbf{p}_{\mathbf{c}}=\left[x_{c} y_{c}\right]$ and characteristic matrix $\mathbf{M}$ is defined by all points $\mathbf{p}=[x y]$ such that

$$
\left(\mathbf{p}-\mathbf{p}_{c}\right)^{T} \mathbf{M}^{-1}\left(\mathbf{p}-\mathbf{p}_{\mathbf{c}}\right) \leq 1 .
$$


The characteristic matrix $\mathbf{M}$ of the ellipse can be written as

$$
\mathbf{M}=\left[\begin{array}{cc}
\cos \theta & -\sin \theta \\
\sin \theta & \cos \theta
\end{array}\right]\left[\begin{array}{cc}
a^{2} & 0 \\
0 & b^{2}
\end{array}\right]\left[\begin{array}{cc}
\cos \theta & -\sin \theta \\
\sin \theta & \cos \theta
\end{array}\right]^{T}
$$

where the parameters of the ellipse are

- $\theta$ the angle between the first axis and the horizontal;

- $a$ the length of the first axis of the ellipse;

- $b$ the length of the second axis of the ellipse;

- $\mathcal{A}$ the area of the ellipse, equal to $\pi a b$.

B. Dynamic model of the virtual structure

The dynamical evolution of the ellipse is modeled by

$\left[\begin{array}{c}x_{c} \\ y_{c} \\ v_{c} \\ \alpha_{c} \\ \theta \\ a \\ b\end{array}\right]_{t+1}=\left[\begin{array}{c}x_{c} \\ y_{c} \\ v_{c} \\ \alpha_{c} \\ \theta \\ a \\ b\end{array}\right]_{t}+\left[\begin{array}{c}d t \cdot v_{c, t} \cdot \cos \left(\alpha_{c, t}\right) \\ d t \cdot v_{c, t} \cdot \sin \left(\alpha_{c, t}\right) \\ 0 \\ 0 \\ 0 \\ 0 \\ 0\end{array}\right]+d t \cdot\left[\begin{array}{c}0 \\ 0 \\ u_{v} \\ u_{\alpha} \\ u_{\theta} \\ u_{a} \\ u_{b}\end{array}\right]$

with

$$
\begin{gathered}
u_{v-} \leq u_{v} \leq u_{v+}, u_{\alpha-} \leq u_{\alpha} \leq u_{\alpha+} \\
u_{\theta-} \leq u_{\theta} \leq u_{\theta+}, u_{a-} \leq u_{a} \leq u_{a+}, \\
u_{b-} \leq u_{b} \leq u_{b+},
\end{gathered}
$$

where $x_{c}, y_{c}, \theta, a, b$ are the aforementioned parameters of the ellipse, $v_{c}$ is the speed of the center and $\alpha_{c}$ its orientation (see Figure 1). The control inputs $u_{v}, u_{\alpha}$ govern the movement of the center of the ellipse $\mathbf{p}_{c}$ by acting on its speed and angular velocity, while the control inputs $u_{\theta}, u_{a}, u_{b}$ modify the characteristic matrix $\mathbf{M}$ (shape and orientation).

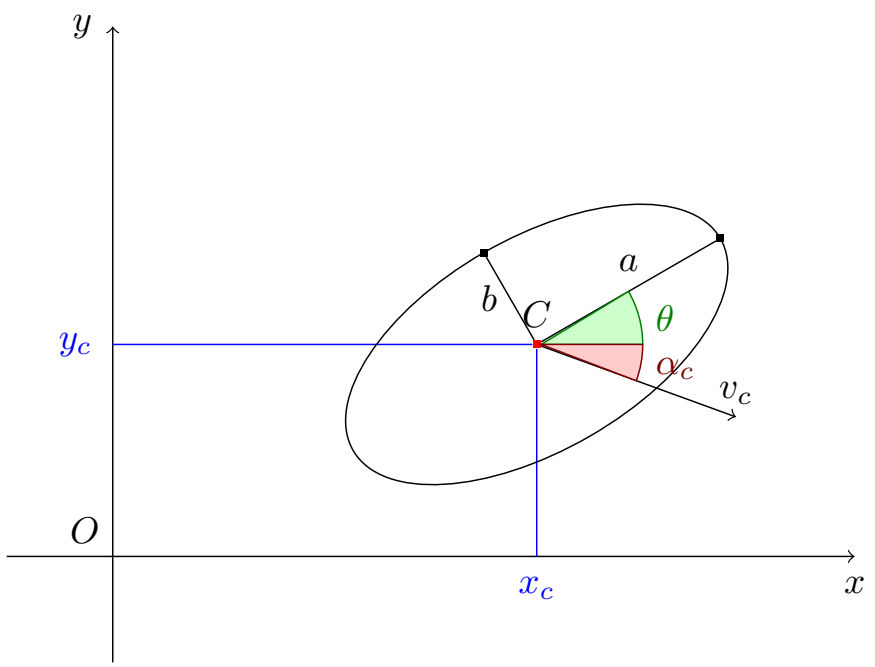

Fig. 1. Ellipse parametrization

This dynamical model should be related to the dynamics of the UAVs so that it does not scatter the formation. The control inputs must thus be selected within suitable range and suitable dynamics. This is yet a clear advantage over methods that modify abruptly the virtual structure and, as a result, do not take into account UAV constraints.

\section{Guidance law design}

A cost function is defined to evaluate how well the virtual structure respects the goals of the mission and the associated constraints. Since collision avoidance should be anticipated and that a dynamical model of the structure is available, it has been chosen to use Model Predictive Control (MPC) to find the optimal control inputs. MPC has already been employed to control fleets of UAVs, e.g., in [16], [17].

MPC predicts the future states of the system on a prediction horizon $H P$ with a sequence of control inputs on a control horizon $H C$, which may be shorter than $H P$. At time $t$, it is possible to compute the $H P$ futures states of the system with the $H C$ control inputs to be sent to the system. The benefit of the combinations of control inputs can then be evaluated with respect to the cost function and the optimal sequence can be found accordingly. Only the first control input vector is then applied to the real system and the MPC scheme is repeated at the next time step [18].

The cost function is composed of terms dealing with the mission objectives and the constraints on the structure itself. The optimal control inputs at time $t$ should minimize the cost function $J_{z}$ such that

$$
\widehat{u}_{\theta}, \widehat{u}_{a}, \widehat{u}_{b}, \widehat{u}_{v}, \widehat{u}_{\alpha}=\arg \min _{\substack{u_{v}, u_{\alpha}, u_{\theta}, u_{a}, u_{b},}} J_{z},
$$

where

$$
J_{z}=J_{\text {target }}+J_{v}+J_{a b}+J_{a b m i n}+J_{c} \text {. }
$$

The components of $J_{z}$ are designed such that

- $J_{\text {target }}$ drives the ellipse to its target;

- $J_{v}$ keeps the ellipse area close to the initial one, $\mathcal{A}$;

- $J_{a b}$ keeps $a$ and $b$ near their initial values $a_{0}$ and $b_{0}$;

- Jabmin maintains $a$ and $b$ bigger than a boundary value so as to avoid the flattening of the ellipse along one of its axes;

- $J_{c}$ modifies the matrix $\mathbf{M}$ to avoid obstacles.

The component $J_{\text {target }}$ is a goal criterion, the others are penalized constraints criteria. This should allow finding values for the control inputs that achieve an acceptable trade-off between the criteria, while hard constraints might not have been verified. Note that the relative weights $w_{(\cdot)}$ of the different contributions should be carefully selected (see Section IV).

1) Target criterion:

$$
J_{\text {target }}=w_{\text {target }} \sum_{k=1}^{H P}\left(\left\|\hat{\mathbf{p}}_{c, t+k}-\mathbf{p}_{\text {target }}\right\|_{2}\right),
$$

where $\hat{\mathbf{p}}_{c, t+k}=\left[\begin{array}{ll}\hat{x}_{c, t+k} & \hat{y}_{c, t+k}\end{array}\right]$ denotes the measured or predicted value of $\hat{\mathbf{p}}_{c}$ at time $t+k$. This notation will be used for all the state variables in the following.

2) Ellipse constraints:

$$
J_{v}=w_{v} \sum_{k=1}^{H P}\left(\hat{a}_{t+k} \cdot \hat{b}_{t+k} \cdot \pi-\mathcal{A}\right)
$$




$$
\begin{gathered}
J_{a b}=w_{a b} \sum_{k=1}^{H P}\left(\left|\hat{a}_{t+k}-a_{0}\right|+\left|\hat{b}_{t+k}-b_{0}\right|\right), \\
J_{\text {abmin }}=w_{\text {abmin }} \sum_{k=1}^{H P} f\left(\hat{a}_{t+k}, l_{1}, l_{2}\right)+f\left(\hat{b}_{t+k}, l_{1}, l_{2}\right),
\end{gathered}
$$

where $f\left(\hat{a}_{t+k}, l_{1}, l_{2}\right)$ is a continuous function, and $l_{1}$ and $l_{2}$ are user-defined parameters, such that

- $f$ takes the value 1 when $\hat{a}_{t+k} \leq l_{1}$,

- $f$ takes the value 0 when $\hat{a}_{t+k} \geq l_{1}+l_{2}$,

- $f$ has a continuous evolution between those two extremal values.

For example, an appropriate choice for $f$ is the function presented in Figure 2.

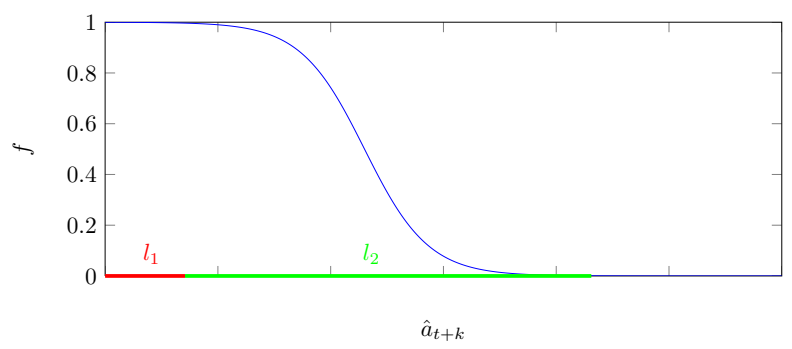

Fig. 2. Function $f$

3) Obstacle avoidance: The structure should maneuver to avoid collision with obstacles of the environment. Assuming that the obstacles are described as convex surfaces (volumes in a 3D case), the intersection area between the virtual structure and the obstacles is computed to detect and quantify potential collisions (Figure 3). Using this value as a penalization in the criterion makes it possible to find a path that minimizes this intersection and thus the risk of possible collision.

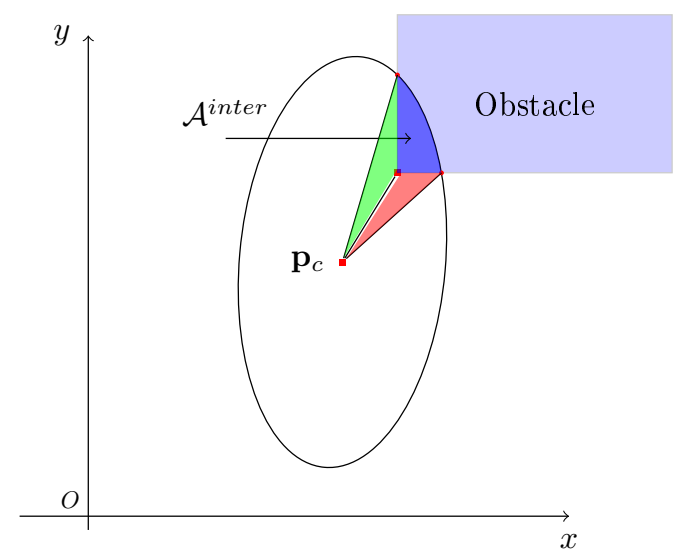

Fig. 3. Ellipse-obstacle intersection

The collision avoidance term $J_{c}$ uses the intersection area $\mathcal{A}_{l, t+k}^{\text {inter }}$ at time $t+k$ for each obstacle $l(\mathrm{NbO}$ being the number of obstacles in the neighborhood). The weight is chosen to give greater importance to the first prediction steps rather than the future ones.

$$
J_{c}=w_{c} \sum_{k=1}^{H P} \sum_{l=1}^{N b O} \frac{H P-k}{H P} \cdot \mathcal{A}_{l, t+k}^{i n t e r}
$$

\section{UAV CONTROL}

The UAV control layer computes the movement of each vehicle so that it remains within the ellipse and avoids collision with the other vehicles. It has thus three goals:

- attract the UAV inside the area

- allocate each UAV inside the area

- avoid collision between UAVs

This control is decentralized (each UAV determines its own control inputs), yet it uses the prediction of the future state of the virtual structure, which is available thanks to the developments from Section II. MPC is used again, since allocation and collision avoidance may benefit from a prediction of the impact of control inputs on the future states of the vehicles. The communication delays and broadcast ranges are ignored for this preliminary research, which means that all the UAVs are assumed to have access without delay to the exact state of all the other vehicles at each time step.

\section{A. Dynamic model of the UAVs}

The $N$ UAVs are assumed to be identical. The state vector of each vehicle $i$ at time $t$ is defined as

$$
\mathbf{x}_{t}^{i}=\left[\begin{array}{c}
x \\
y \\
v \\
\alpha
\end{array}\right]_{i, t}
$$

where $\left[\begin{array}{ll}x y & y\end{array}\right]$ is the position of the UAV, $v$ its speed and $\alpha$ its orientation. Faster dynamics (e.g., autopilot) are neglected for guidance law determination. The dynamical model in discrete time is

$$
\begin{gathered}
{\left[\begin{array}{l}
x \\
y \\
v \\
\alpha
\end{array}\right]_{i, t+1}=\left[\begin{array}{l}
x \\
y \\
v \\
\alpha
\end{array}\right]_{i, t}+d t\left[\begin{array}{c}
v_{i, t} \cos \left(\alpha_{i, t}\right) \\
v_{i, t} \sin \left(\alpha_{i, t}\right) \\
0 \\
0
\end{array}\right]+d t\left[\begin{array}{c}
0 \\
0 \\
u_{1, i, t} \\
u_{2, i, t}
\end{array}\right]} \\
\text { within constraints : }\left\{\begin{array}{l}
v_{\min } \leq v_{i, t+1} \leq v_{\max } \\
\left|u_{2}, i, t\right| \leq d \alpha_{\max }
\end{array}\right.
\end{gathered}
$$

For each UAV (index $i$ is now omitted), the control inputs $u_{1, t}$ and $u_{2, t}$ should be determined at each time $t$ such that

$$
\widehat{u}_{1}, \widehat{u}_{2}=\arg \min _{u_{1}, u_{2}} J_{d}
$$

where

$$
J_{d}=J_{t}+J_{c o l}+J_{n 1}+J_{n 2}+J_{u}
$$

The components of $J_{d}$ are designed such that

- $J_{t}$ drives the UAV inside the area;

- $J_{c o l}$ modifies the direction and the speed to avoid collision with others UAVs; 
- $J_{n 1}$ keeps the speed of the UAV close to a chosen value;

- $J_{n 2}$ keeps the orientation of the UAV close to the one of the structure;

- $J_{u}$ minimizes the energy consumption in terms of control inputs.

1) Attraction and allocation of the UAVs inside the structure: The Mahalanobis distance [19] evaluates the norm between a point and an area (e.g., the ellipse considered here) defined by its center and characteristic matrix. This makes it possible to define a norm weighted by the size and the orientation of the ellipse. The Mahalanobis distance (Figure 4) of a point $\mathbf{p}=\left[\begin{array}{ll}x & y\end{array}\right]^{T}$ to the ellipse defined by its center $\mathbf{p}_{c}=\left[\begin{array}{ll}x_{c} & y_{c}\end{array}\right]^{T}$ and its matrix $M$, is given by

$$
d_{M}(\mathbf{p})=\sqrt{\left(\mathbf{p}-\mathbf{p}_{c}\right)^{T} \mathbf{M}\left(\mathbf{p}-\mathbf{p}_{c}\right)} .
$$

The term $J_{t}$ is used to lead the UAVs inside the virtual structure. The Mahalanobis distance is employed to reflect the shape of the ellipse.

$$
J_{t}=w_{t} \sum_{k=1} \frac{(H P-k)}{H P} g(i, k),
$$

where the function $g$ is defined as

$$
g(i, k)=\left\{\begin{array}{c}
\bullet d_{M, k}\left(\hat{\mathbf{x}}[1: 2]_{i, t+k}\right) \\
\text { if } \quad d_{M, k}\left(\hat{\mathbf{x}}[1: 2]_{i, t+k}\right)>1 \\
\bullet d_{M, k}\left(\hat{\mathbf{x}}[1: 2]_{i, t+k}\right)-1 \\
\text { if } d_{M, k}\left(\hat{\mathbf{x}}[1: 2]_{i, t+k}\right)<1
\end{array}\right.
$$

The weight $(H P-k) / H P$ is meant to give more importance to the first predictions than the later ones. The function $g(i, k)$ is built on the basis of the Mahalanobis distance of the UAV position to the ellipse center. This function introduces a potential field that guides the UAV inside the area. A discontinuity has been added to make a stronger difference at the boundary of the virtual structure. A projection of function $g$ is provided in Figure 5.

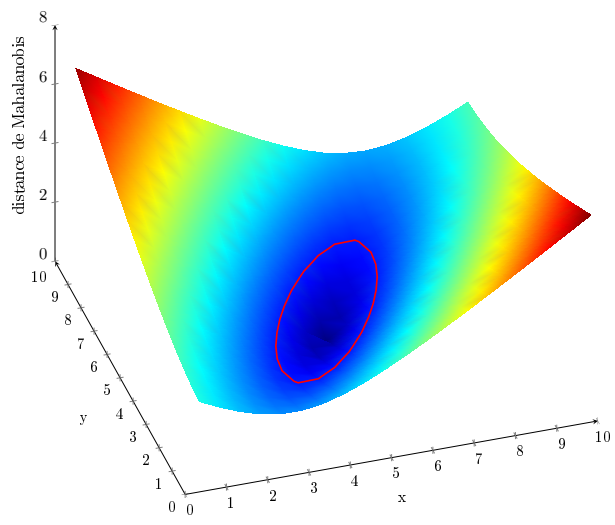

Fig. 4. Mahalanobis distance over position space, ellipse in red

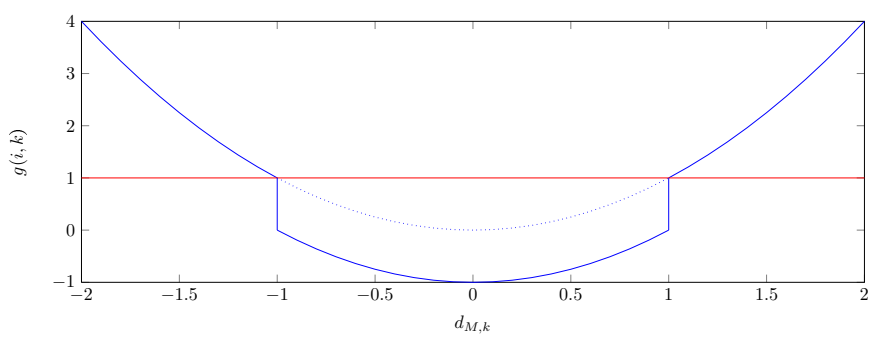

Fig. 5. Aspect of the function $g$ (2D projection)

2) Collision avoidance: The term $J_{c o l}$ is used to avoid collision between UAVs. Two distances are defined, namely $d_{1}$ a safety distance and $d_{2}$ an influence distance. The objective is to maintain the distance between UAVs larger than $d_{1}+d_{2}$ and to forbid it to be smaller than $d_{1}$. The criterion $J_{c o l}$ should be null when the distance between two UAVs is bigger than $d_{1}+d_{2}$, and it should be maximum when this distance becomes smaller than $d_{1}$. A continuous evolution is preferable between those two extremal values. The weighting function $f$ presented in Figure 2 is appropriate for this purpose, its parameters being here $d_{i, j, k}$ (the distance between the two UAVs considered), $d_{1}$ and $d_{2}$ with

$$
\begin{gathered}
d_{i, j, k}=\left\|\left(\hat{\mathbf{x}}[1: 2]_{i, t+k}-\mathbf{x}[1: 2]_{j, t}\right)\right\|_{2} . \\
J_{c o l}=w_{c o l} \sum_{\substack{j=1 \\
j \neq i}}^{N} \sum_{k=1}^{H P} f\left(d_{i, j, k}, d_{1}, d_{2}\right)
\end{gathered}
$$

3) Formation consistency: The component $J_{u}$ is used to minimize the magnitude of the control inputs sent to the UAV.

$$
J_{u}=\frac{1}{H C} \sum_{k=1}^{H C}\left(u_{1, i, t+k}^{2}+u_{2, i, t+k}^{2}\right)
$$

The components $J_{n 1}$ and $J_{n 2}$ are used to keep the speed of the UAV close to a value $v_{0}$ and the orientation of the UAV close to the one of the ellipse $\alpha_{c}$.

$$
\begin{gathered}
J_{n 1}=w_{n 1} \sum_{j=1}^{H P}\left(\hat{\mathbf{x}}[3]_{i, t+j}-v_{0}\right)^{2} \\
J_{n 2}=\frac{w_{n 2}^{0}}{H P} \sum_{k=1}^{H P} w_{n 2}^{k} \cdot\left(\hat{\mathbf{x}}[4]_{i, t+k}-\alpha_{c}\right)^{2}
\end{gathered}
$$

The weights $w_{n 2}^{k}$ depend on the Mahalanobis distance between the UAV and the ellipse. When the distance is less than a value $l_{3}$, then $w_{n 2}^{k}$ is equal to one and when it's bigger than $l_{4}, w_{n 2}^{k}$ is equal to 0 . The function $f$ (Figure 2) is used again.

$$
w_{n 2}^{k}=f\left(d_{M, k}, l_{3}, l_{4}\right)
$$




\section{Simulation Results}

\section{A. Simulation description}

A simple scenario has been defined to test the guidance law. The formation should reach a predefined target $\mathbf{p}_{\text {target }}$ starting from $\mathbf{p}_{c, 0}$ with an initial orientation of the ellipse perpendicular to the movement. Two rectangular obstacles cross the trajectory of the formation. To avoid collision with these obstacles, the virtual structure will have to modify its shape so as to pass the obstacles safely. Only the deformation is considered here $\left(u_{\theta}=0\right)$, but an additional rotation of the structure could be handled similarly.

\section{B. Weight values}

The values of the weights for the ellipse control (higher layer) are chosen as follows:

- $w_{\text {target }}=10^{-2}$;

- $w_{v}=10^{-4}$;

- $w_{a b}=10$

- $w_{\text {abmin }}=10^{-3}$;

- $w_{c}=100$.

The weights $w_{\text {target }}, w_{v}$ and $w_{\text {abmin }}$ are small so as to normalize the corresponding components $J_{\text {target }} J_{v}$ and $J_{a b m i n}$ that may reach high values compared to the others. $J_{a b}$ takes values only between 0 and 1 , so $w_{a b}$ should take a more important value. The weight $w_{c}$ has a stronger value since the obstacle avoidance constraint should take precedence over the other components.

The weight values for the UAV control layer are

- $w_{t}=100$;

- $w_{\text {col }}=100$;

- $w_{n 1}=1$;

- $w_{n 2}^{0}=10$.

$w_{n 1}$ is the smallest weight since speed control is not the most important term. $w_{n 2}^{0}$ has a bigger value because the orientation is deemed to be more important than speed control. $w_{t}$ and $w_{c}$ have the biggest values because $J_{t}$ and $J_{c}$ are the criteria for collision avoidance and formation organization, which are the most important goals the UAVs should fulfill.

\section{Parameter values}

The initialization parameters of the simulation are given in Table I. Note that the virtual structure has a longer prediction horizon than the UAVs, since it holds more information on the final destination and the target.

\section{TABLE I}

SIMULATION PARAMETERS

\begin{tabular}{|c|c|c|c|}
\hline$N=8$ & $v_{0}=4$ & $v_{\min }=2$ & $v_{\max }=6$ \\
\hline$d \alpha_{\text {max }}=0.3$ & $d t=1$ & $d_{1}=6$ & $d_{2}=40$ \\
\hline$H P_{\text {uav }}=10$ & $H C_{\text {uav }}=5$ & $H P_{\text {ell }}=30$ & $H C_{\text {ell }}=5$ \\
\hline$l_{1}=70$ & $l_{2}=90$ & $l_{3}=5$ & $l_{4}=10$ \\
\hline$a_{0}=200$ & $b_{0}=100$ & $v_{c}=4$ & $N b O=2$ \\
\hline$\alpha_{c, 0}=\pi / 2$ & $\mathbf{p}_{c, 0}=\left[\begin{array}{lll}100 & 0\end{array}\right]$ & $\mathbf{p}_{\text {target }}=\left[\begin{array}{lll}2000 & 0\end{array}\right]$ & \\
\hline
\end{tabular}

\section{Results}

An example of gathering of the UAVs inside the virtual structure is displayed in Figure 6.

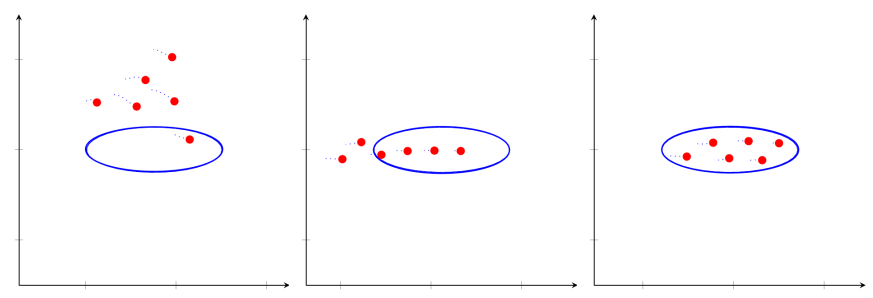

Fig. 6. Gathering of UAVs within the ellipsoidal structure
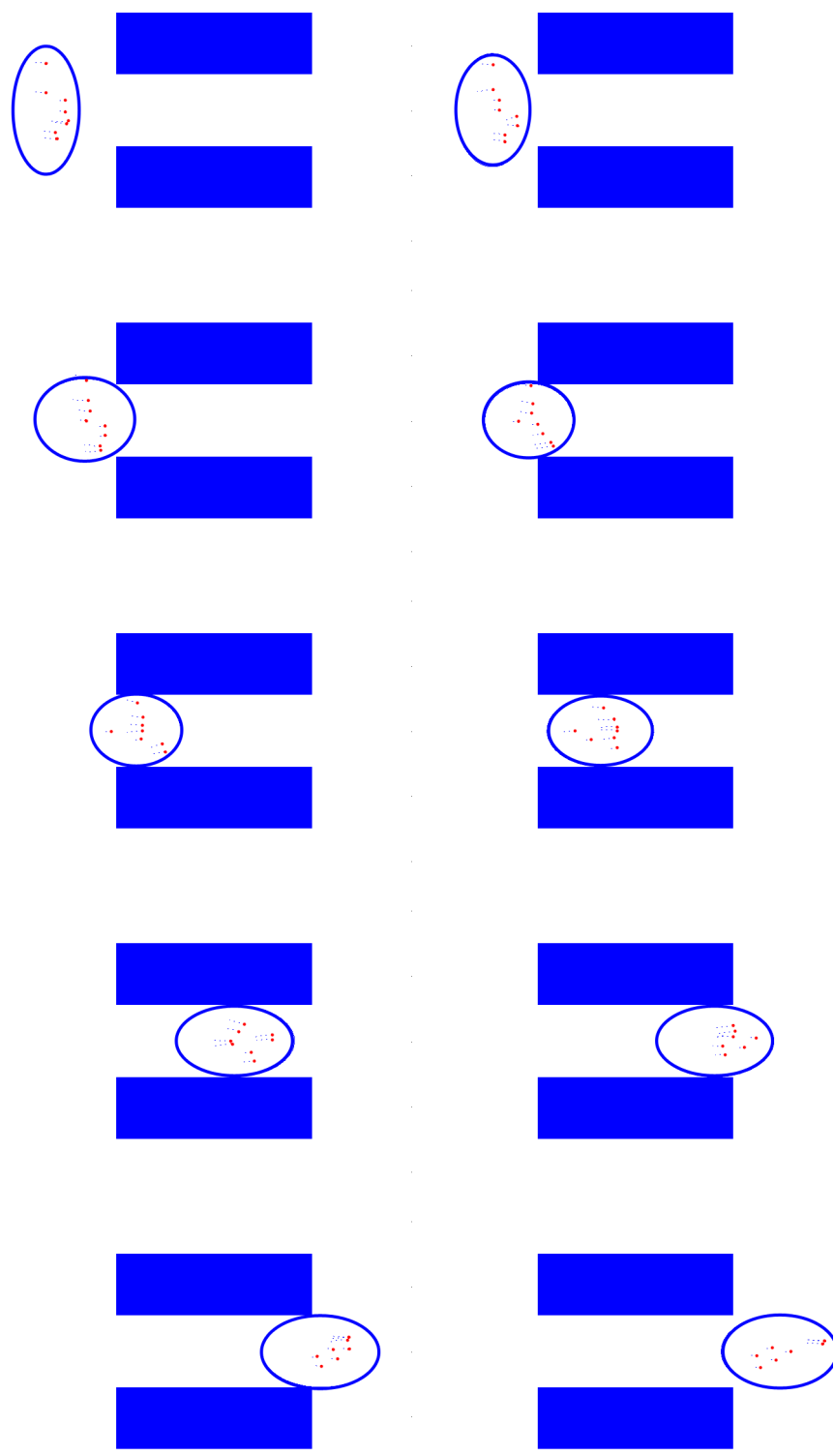

Fig. 7. Obstacle avoidance by deformation 
The complete scenario is illustrated by the sequence in Figure 7. The ellipse modifies its shape accordingly when approaching the obstacles and no collision has been reported. The UAVs were initially in a vertical formation inside the ellipse. When the ellipse changes, the formation is modified to keep all the UAVs inside the structure. It can be seen that, since the range of the ellipse control inputs have been chosen to cope with the UAV dynamics, the vehicles have sufficient time to remain inside the virtual structure when it is modified. The area of the ellipse is also kept close to its initial value. Figure 8 shows the values of the control inputs $u_{a}$ and $u_{b}$ that govern the deformation of the structure over time. These input values modify the length of the two axes simultaneously and almost symmetrically to cope with the area constraint.

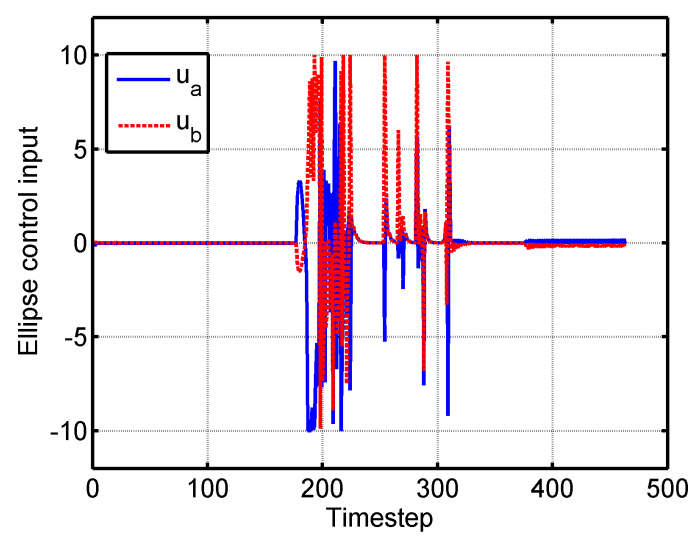

Fig. 8. Control inputs for ellipse deformation

The proposed method thus makes it possible to maintain the UAVs inside an elliptical virtual structure with collision avoidance, thanks to the two-layer guidance law. The higher layer modifies the characteristics of the virtual structure with only knowledge of the obstacles and target while the lower layer modifies the formation and repartition of the UAVs in a decentralized way based only on the knowledge of the actions from the upper layer.

\section{CONCLUSiOnS AND PERSPECTIVES}

In this paper, a two layer guidance law has been presented for formation flight with obstacle avoidance. An elliptical virtual structure is used to give its shape to the formation and modify it to cope with obstacles of the environment. The UAVs are then attracted inside the structure and avoid collision between themselves. Model predictive control has been selected to achieve the goals of these two layers.

Simulation results have shown the interest of this strategy for formation flying. The main novelty is the modification of the virtual structure thanks to its dynamics, which results in a change of geometry for the formation. This modification is useful to avoid collisions with obstacles by reconfiguring the fleet of UAVs.

Other shapes for the virtual structure could be taken into account inside this guidance scheme by modifying the dy- namical model of the structure, some of the criteria and the Mahalanobis distance. Future work will also focus on the adaptation of this scheme to non-convex obstacles. A possible solution would be to divide the virtual structure into sub-structures that are able to bypass the obstacles without collision.

\section{REFERENCES}

[1] J. Desai, J. Ostrowski, and V. Kumar, "Controlling formations of multiple mobile robots," Proceedings of the IEEE International Conference on Robotics and Automation, Leuven, Belgium, vol. 4, no. May, pp. 2864-2869, 1998

[2] H. Tanner and G. Pappas, "Leader-to-formation stability," IEEE Transactions on Robotics and Automation, vol. 20, no. 3, pp. 443-455, 2004

[3] A. Jadbabaie and J. Lin, "Coordination of Groups of Mobile Autonomous Agents Using Nearest Neighbor Rules," Proceedings of the 41st IEEE Conference on Decision and Control, Las Vegas, USA, vol. 48, no. 6, pp. 988-1001, 2003.

[4] T. Vicsek, A. Czirók, E. Ben-Jacob, I. Cohen, and O. Shochet, "Novel type of phase transition in a system of self-driven particles," Physical Review Letters, vol. 75, no. 6, pp. 4-7, 1995.

[5] C. Reynolds, "Flocks, herds and schools: A distributed behavioral model," ACM SIGGRAPH Computer Graphics, vol. 21, no. 4, 1987.

[6] S. Carpin and L. Parker, "Cooperative leader following in a distributed multi-robot system," in Proceedings of the IEEE International Conference on Robotics and Automation, Washington D.C., USA, vol. 3, 2002, pp. 2994-3001.

[7] Q. Chen and J. Luh, "Coordination and control of a group of small mobile robots," IEEE Transactions on Robotics and Automation, vol. 3, pp. 2315-2320, 1994.

[8] L. Parker, "ALLIANCE: An architecture for fault tolerant multirobot cooperation," IEEE Transactions on Robotics and Automation, vol. 14, no. 2, pp. 220-240, 1998.

[9] T. Balch and R. Arkin, "Behavior-based formation control for multirobot teams," IEEE Transactions on Robotics and Automation, vol. 14, no. 6 pp. 926-939, 1998.

[10] M. Schneider-Fontan and M. Mataric, "Territorial multi-robot task division," IEEE Transactions on Robotics and Automation, vol. 14, no. 5, pp. 120-124, 1998.

[11] L. Parker, "Decision Making as Optimization in Multi-robot Teams," in Proceedings of 8th International Conference on Distributed Computing and Internet Technology, Bhubaneswar, India, 2012, pp. 35-49.

[12] M. Lewis, "Virtual structures for high-precision cooperative mobile robotic control," Proceedings of IEEE/RSJ International Conference on Intelligent Robots and Systems, Osaka, Japan, vol. 1, pp. 132-139, 1996.

[13] N. Leonard," "Virtual leaders, artificial potentials and coordinated control of groups," Proceedings of the IEEE Conference on Decision and Control, Orlando, USA, pp. 2968-2973, 2001.

[14] L. Barnes, M. Fields, and K. Valavanis, "Swarm formation control utilizing elliptical surfaces and limiting functions." IEEE transactions on systems, man, and cybernetics. Part B, Cybernetics, vol. 39, no. 6, pp. 1434-45, 2009.

[15] M. Bacon, "Robust region tracking for swarms via a novel utilization of sliding mode control," Intelligent Control and Automation, vol. 3, no. 1, pp. 98-109, 2012.

[16] R. Saber, W. Dunbar, and R. Murray, "Cooperative control of multivehicle systems using cost graphs and optimization," in Proceedings of the American Control Conference, Denver, USA, vol. 3, 2003, pp. 22172222.

[17] Y. Rochefort, S. Bertrand, H. Piet-Lahanier, D. Beauvois, and D. Dumur, "Cooperative Nonlinear Model Predictive Control for Flocks of Vehicles," IFAC Workshop on Embedded Guidance, Navigation and Control in Aerospace, Bangalore, India, 2012.

[18] R. Findeisen, L. Imsland, F. Allgower, and B. A. Foss, "State and output feedback nonlinear model predictive control: An overview," European Journal of Control, vol. 9, no. 2-3, pp. 190-206, 2003.

[19] R. D. Maesschalck, D. Jouan-Rimbaud, and D. Massart, "The Mahalanobis distance," Chemometrics and Intelligent Laboratory Systems, vol. 50, no. 1, pp. 1-18, 2000. 